\title{
Anatomy of the Inferior Alveolar Nerve in Rabbits and its Block Anesthesia
}

\author{
Yong-Di Li, Zheng-Long Tang*, Jian-Qin Tan, Dong-Xiang WANG and You-Li Chen
}

Department of Oral and Maxillofacial Surgery, Affiliated Hospital of Guizhou Medical University, China

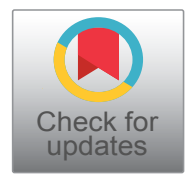

*Corresponding author: Zheng-Long Tang, Department of Oral and Maxillofacial Surgery, Affiliated Hospital of Guizhou Medical University, Guiyang, China, Tel: +86-0851-86773504, Fax: 86-0851-6772901, Postal Code: 550004, E-mail: zhenglongtang@hotmail.com

\begin{abstract}
Objective: This study performed an anatomical investigation of the mandible and inferior alveolar nerve in rabbits to provide an anatomical basis for the establishment of animal models for mandibular experiments and the implementation of block anesthesia in the inferior alveolar nerve.

Methods: The mandible and the inferior alveolar nerve were dissected in 10 rabbits to investigate and measure the traveling distribution of the inferior alveolar nerve. A total of 20 rabbits were divided into an experimental group and a control group. A mandibular distraction osteogenesis model was established. Block anesthesia for the inferior alveolar nerve was conducted by the extraoral method, and anesthetic efficacy was evaluated.

Results: The inferior alveolar nerve in rabbits enters the mandibular canal at the neural foramen of the medial mandibular ascending branch and is distributed in the mandible. The vertical distance between the anterior incisure of the mandibular angle and the mandibular neural foramen is 10-11 mm. When block anesthesia was performed on the inferior alveolar nerve at the anterior incisure of the mandibular angle, the rabbits in the experimental group showed a significantly lower degree of pain than those in the control group.

Conclusion: The inferior alveolar nerve in rabbits is a sensory nerve that travels in the mandibular canal and is distributed on the mandible and mandibular teeth. Block anesthesia of the inferior alveolar nerve through the lower edge of the mandible is a simple and straightforward operation with an enhanced anesthesia efficacy for mandibular surgery in rabbits.
\end{abstract}

\section{Keywords}

Rabbit, Mandible, Inferior alveolar nerve, Anatomy, Nerve block anesthesia
Rabbits are commonly used as experimental animals in the study of oral and maxillofacial diseases. Experimental studies of mandibular defect repair, mandibular distraction osteogenesis, inferior alveolar nerve damage, dental implant, and orthodontic tooth movement are often based on models of the mandible and mandibular teeth in rabbits [1-9]. Anesthesia is critical to ensure the establishment of animal models and the smooth implementation of the experiment. Successful anesthesia should meet the requirements of intraoperative sedation and painlessness and minimize the chance of anesthesia accidents to ensure experimental success. Currently, rabbits are usually anesthetized by intravenous or intraperitoneal injection during experiments, but unsatisfactory analgesic effects or death due to overdose of the anesthesia agents during surgery can sometimes occur in experimental rabbits. For mandibular experiments in rabbits, combining general anesthesia with local anesthesia may achieve a better analgesic effect. The application of local infiltration anesthesia at the surgical site is simple, but the analgesic effect may be reduced due to the separation of the tissue flap and local dilution. Ogawa, et al. [10] found that following local infiltration anesthesia of the mandible using lidocaine, the concentration of lidocaine at the mandible was decreased due to stripping in the periosteum of the mandible and the dilution by saline, resulting in a reduced anesthetic effect. Thus, for long-lasting mandible surgery, general anesthesia vial local nerve block anesthesia may offer the highest analgesic effect. The distribution of nerves in the surgical area needs to be 
clarified in order to perform nerve block anesthesia, as the local anesthetic agent must be precisely injected at the position of the neural stem to achieve anesthesia. Campillo, et al. [11] performed an anatomical and morphological study of the rabbit mandible, but the traveling distribution of the inferior alveolar nerve in rabbits and its block anesthesia are not yet fully understood.

The present study reports the dissection of the mandible and inferior alveolar nerve in rabbits and investigates the extraoral block anesthesia of the inferior alveolar nerve, as well as its efficacy in surgery for mandibular distraction osteogenesis in rabbits, which is expected to provide an anatomical basis and block anesthesia approach to establish animal models for mandible experiments in rabbits.

\section{Materials and Methods}

\section{Experimental animals}

This study used 30 specific pathogen free (SPF) New Zealand white rabbits (both male and female) that were 6 -months-old and weighed $2.0 \pm 0.1 \mathrm{~kg}$. The code of the Animal Certificate of Conformity is SCXK (Chongqing) 2007-0005. After they were purchased, the rabbits were housed separately in the Experimental Animal Center of Guizhou Medical University. Standard feed was provided to each rabbit in the form of $350 \mathrm{~g}$ of pellets per day and running tap water was freely accessible to drink at any time. The experimental program was approved by the Laboratory Animal Ethics Committee of Guizhou Medical University.

\section{Experimental equipment}

The experimental equipment included a set of surgical instruments, an operating table for rabbits, gauze, a fixed box, beakers, and a ruler.

\section{Dissection of the mandible}

Each animal was sacrificed with an overdose of $3 \%$ sodium pentobarbital. The coated hair around the jaw of the rabbit was shaved to expose the skin. A 6-cm incision was created using a scalpel on the skin along the lower edge of one side of the mandible. The soft tissue around the mandible and the mandibular condyle were dissected layer by layer to completely separate the mandible as an intact piece. The length of the mandible was measured, as were the distance and angle of the neural foramen of the inferior alveolar nerve entering the mandible to the surrounding anatomical landmarks.

\section{Dissection to expose the inferior alveolar nerve}

The soft tissue on the inner side of the mandible was stripped with a periosteal stripper to reveal the mandibular foramen. After the inferior alveolar nerve was exposed, the separation was continued towards the base of the skull. Along the traveling direction of the inferior alveolar nerve, the bone in the medial mandible was carefully removed with an osteotome, exposing the inferior alveolar nerve to the mental foramen. The traveling and distribution of the inferior alveolar nerve were observed and investigated.

\section{Evaluation of the efficacy of block anesthesia of the inferior alveolar nerve}

Grouping: Twenty experimental animals were randomly divided into two groups. The rabbits in the experimental group received general anesthesia plus block anesthesia with lidocaine for the inferior alveolar nerve, whereas the rabbits in the control group received general anesthesia plus anesthesia with saline injection for the inferior alveolar nerve.

Surgery for mandibular distraction osteogenesis: After general anesthesia with 3\% sodium pentobarbital, rabbits were fixed on the operating table to perform the block anesthesia of the inferior alveolar nerve. Block anesthesia was performed as follows: The needle entered at the mandibular incisure towards the medial mandibular ascending branch, with the angle between the needle and the lower edge of the mandible measuring approximately $120^{\circ}$. After the depth of the needle reached approximately $11 \mathrm{~mm}, 5 \mathrm{ml}$ of lidocaine was injected. After the anesthesia started to function, the skin and the subcutaneous tissue were cut open along the lower edge of the mandible to the bone surface. Subperiosteal dissection was performed to expose the outer side of the mandible. Mandibular vertical osteotomy at the mandibular first molar was conducted using a fissure bur, with cooling by saline during the osteotomy. Finally, a distractor was inserted and fixed (Figure 1), followed by layered suture of the incision after hemostasis.

Evaluation of the anesthesia efficacy for the inferior alveolar nerve: Intraoperative pain was classified according to four levels. Level 1: No response; level 2: Showing oral and maxillofacial activities; level 3: Activities in the limbs, with slight struggle; level 4: Systemic violent struggle, with surgery unable to proceed. The data were analyzed using the Mann-Whitney $U$ test, with $p<0.05$ considered statistically significant.

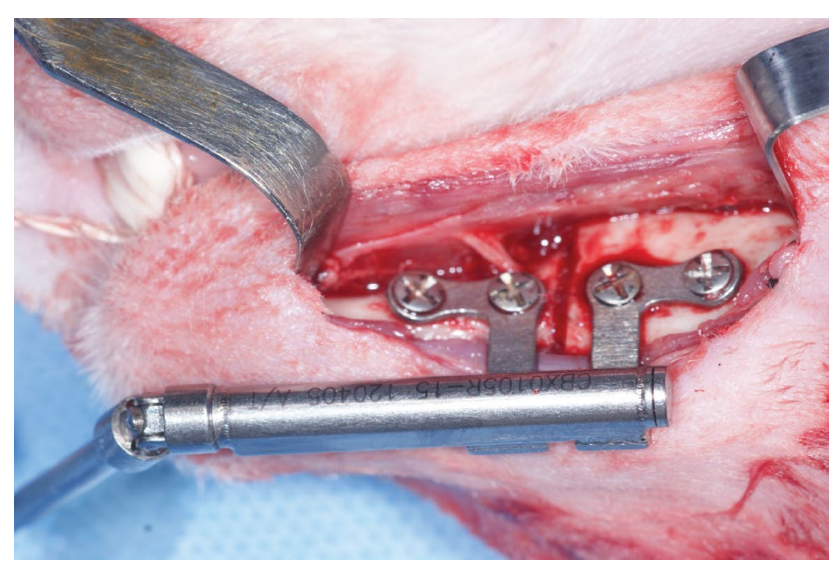

Figure 1: Mandibular vertical osteotomy was conducted, then a distractor was inserted and fixed. 


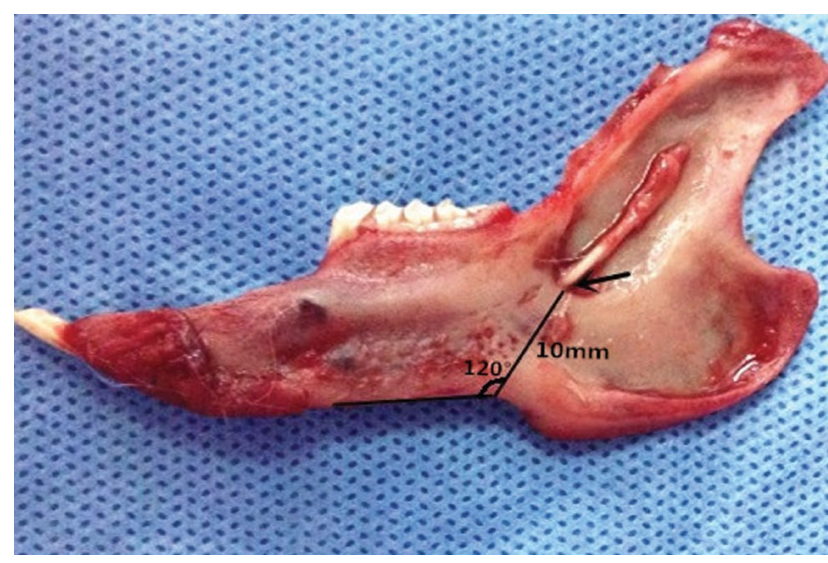

Figure 2: The positional relationship of the mandibular foramen (arrow) and the lower mandibular edge, as well as the incisure of the lower mandibular edge.

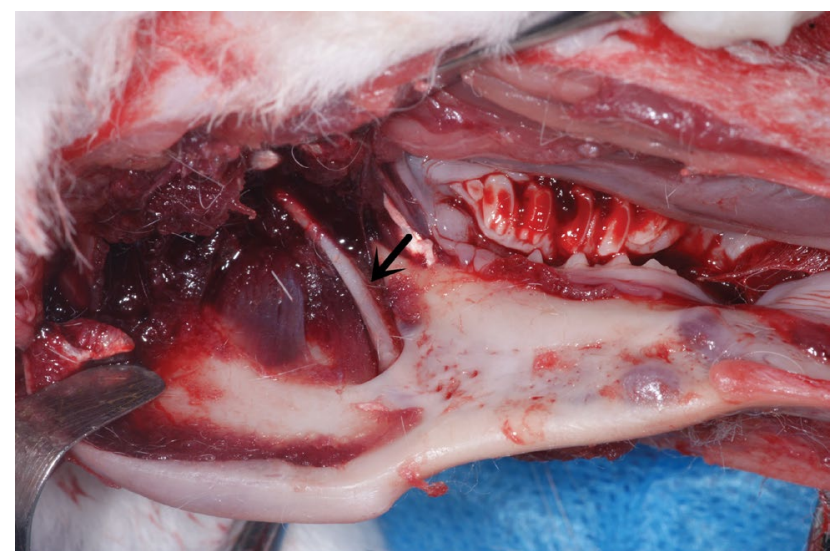

Figure 3: After exiting the skull, the inferior alveolar nerve (arrow) travels between muscles and enters the mandibular foramen.

\section{Results}

\section{Anatomical measurements for the mandible}

A total of 10 mandible specimens were obtained. The average side length of the mandibles was $70 \mathrm{~mm}$ ( $\pm 2 \mathrm{~mm}$ ). In the mandibular ascending branch slightly biased to the root of the $5^{\text {th }}$ molar, the mandibular foramen was observed as a funnel-shape, with the opening towards the upper rear. The vertical distance of the mandibular incisure and the mandibular foramen was approximately 10-11 $\mathrm{mm}$. The angle between the anterior edge of the mandible and the connecting line of the incisure in the lower mandibular edge and the mandibular foramen was approximately $120^{\circ}$ (Figure 2).

\section{Anatomical measurements for the inferior alveolar nerve}

After exiting the skull, the inferior alveolar nerve travels along the medial mandibular ascending branch and the gap between muscles, downwards into the mandibular foramen (Figure 3), and then along the mandibular nerve canal, with branches emitted at various roots and the mandible. The mental nerve was observed on the outer surface of the stripped soft tissue

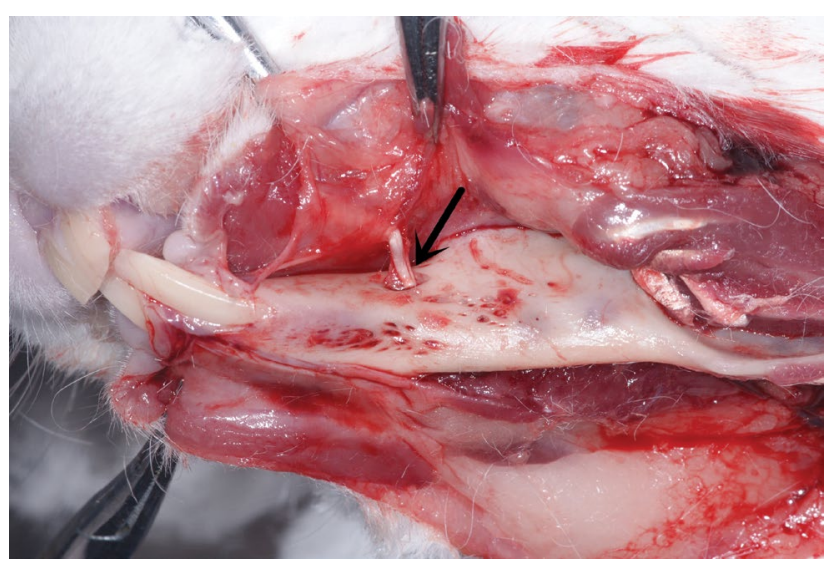

Figure 4: The path of the mental foramen and mental nerve (arrow).

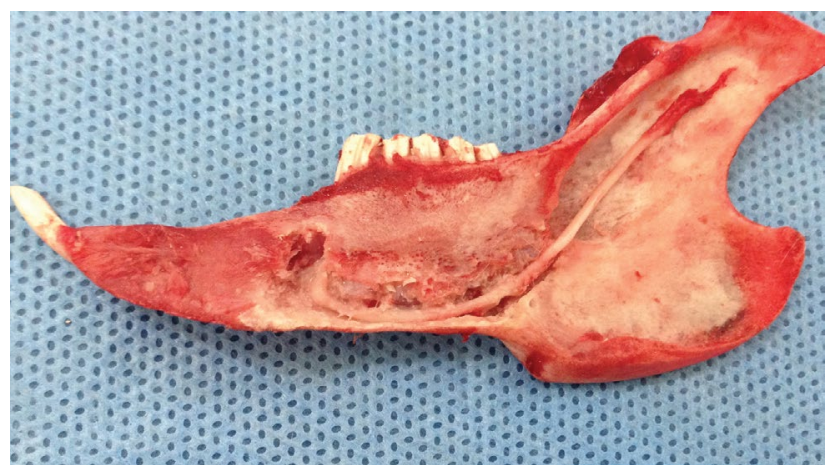

Figure 5: The path and distribution of the inferior alveolar nerve in the mandible (medial view).

and piercing from the mental foramen (Figure 4). The inferior alveolar nerve in the mandibular body is located approximately $1 \mathrm{~mm}$ above the lower edge of the mandible and below the molar root tip, traveling outwards from the mesial site of the first molar to pierce the mental foramen into the periosteum and subcutaneous soft tissues (Figure 5).

\section{Efficacy of the block anesthesia of the inferior al- veolar nerve}

After receiving block anesthesia of the inferior alveolar nerve by $2 \%$ lidocaine hydrochloride injection, the rabbits in the experimental group displayed no signs of intraoperative pain, struggle or contractile response in the limbs and body, and the operation proceeded smoothly. For the animals in the control group, no significant pain occurred when the skin was cut, but obvious signs of pain were observed during mandibular osteotomy and the distractor implantation, with head and neck activities and struggling of the limbs and the body. Additional general anesthesia was often needed to complete the mandible osteotomy and distractor fixation. The difference in the pain level between the two groups was statistically significant (Table 1 ).

\section{Discussion}

Rabbits are similar to humans in some physiologi- 
Table 1: Comparison of the intraoperative pain response.

\begin{tabular}{|l|l|l|l|l|l|l|}
\hline Group & \multicolumn{2}{l|}{ Pain level } & \multicolumn{3}{l|}{ Mann-Whitney U } \\
\cline { 2 - 3 } & Level 1 & Level 2 & Level 3 & Level 4 & Z & P \\
\hline Experimental group & 5 & 4 & 1 & 0 & -3.688 & 0.001 \\
\hline Control group & 0 & 0 & 6 & 4 & & \\
\hline
\end{tabular}

cal functions. In addition, due to their fast propagation and rapid formation of mature bone, rabbits are widely used as animal models for human diseases. Studies of the rabbit mandible and the traveling distribution of its inferior alveolar nerve can provide an anatomical basis for the implementation of effective local anesthesia and the establishment of experimental models. However, the relevant anatomical data on the inferior alveolar nerve in rabbits and the research methods for its block anesthesia are still lacking. The human mandible forms a "U" shape, and block anesthesia for the inferior alveolar nerve is often applied through the mouth during surgery to perform mandibular osteotomy, dental implantation and tooth extraction, with good local analgesic effect. The mandible in rabbits forms a "V" shape, and the mouth is relatively deep, making it difficult to perform block anesthesia for the inferior alveolar nerve through the mouth. Campillo, et al. [11] conducted anatomical and histomorphological studies on the rabbit mandible, and the results showed that the distance from the mandibular first molar to the incisor is $19 \mathrm{~mm}$ in rabbits and that the mental foramen is located on the rear two-thirds of this area, $0.8 \mathrm{~mm}$ from the upper edge and $4.6 \mathrm{~mm}$ from the lower edge of the mandible, in the medial and lateral sides of the mandible, attaching with the masseter muscle and the pterygoid muscle. In the present study, the average side length of mandible in rabbit was found to be $70 \mathrm{~mm}( \pm 2 \mathrm{~mm}$ ), the vertical distance between the incisure in the lower mandibular edge and the mandibular foramen in the medial mandibular ascending branch was approximately 10-11 mm, and the angle between the anterior edge of the mandible and the connecting line of the incisure in the lower mandibular edge and the mandibular foramen was approximately $120^{\circ}$. These findings provide an anatomical database for the implementation of block anesthesia of the inferior alveolar nerve in rabbits.

The present study carried out anatomical measurements of the rabbit mandible, investigated the position of the inferior alveolar nerve foramen and the distribution of the inferior alveolar nerve, and recorded the anatomical features of the mandible and the inferior alveolar nerve in rabbits; the block anesthesia of the inferior alveolar nerve in rabbits, as well as its efficacy, were also explored. Campillo, et al. [11] found that the inferior alveolar nerve in rabbit's travels along the mandibular canal and is located at the root tip of the mandibular dentition in the mandibular canal. The results of the present study showed that the inferior alveolar nerve in rabbits enters the mandibular canal at the neural foramen of the medial mandibular ascending branch and that the branches are basically matched in the mandibular alveolar base, with terminal branches piercing the mental foramen to anastomose with the corresponding contralateral nerve. In the mandible, the inferior alveolar nerve travels below the molar root tip, approximately $1.0 \mathrm{~mm}$ from the lower edge of the mandible. The position of the incisure in the lower mandibular edge in rabbits was also found to be constant and easy to reach, which can serve as a landmark for the extraoral block anesthesia of the inferior alveolar nerve in rabbits. The needle can enter at an approximately $120^{\circ}$ angle relative to the lower mandibular edge, towards the medial mandibular ascending branch. The local anesthetic agent can be injected after the needle reaches a depth of approximately $11 \mathrm{~mm}$ along the bone surface, resulting in the anesthesia in a side of the mandible and teeth.

A variety of anesthesia agents have been used in rabbits, including the anesthetic or sedative drugs sodium pentobarbital, chloral hydrate, ketamine, fentanyl, and sevoflurane, via intravenous injection or inhalation. Although these agents show good sedative effects, higher doses of these anesthetics are needed to achieve a satisfactory analgesic effect. Increasing the dose of anesthetic drugs can increase their toxicity, which tends to increase the anesthetic complications and the mortality of the animals during surgery. The barbiturate sodium pentobarbital is a central nervous system depressant with medium effect. With increasing doses, this compound can induce effects ranging from sedation to hypnosis and anesthesia; an excessive dose may cause respiratory depression, leading to death. The synergistic use of multiple agents can achieve better anesthetic effects but can also cause changes in physiological and biochemical metabolic indicators, as well as drug interactions, which may interfere with the experimental results [12-14]. Therefore, different anesthesia approaches should be applied to rabbit models for different experiments [15-17]. In the present study, the rabbits in the experimental group received intravenous anesthesia through the ear using sodium pentobarbital and block anesthesia of the inferior alveolar nerve in the medial mandibular ascending branch. The experimental animals showed no pain response during mandibular surgery, indicating that the extraoral block anesthesia of the inferior alveolar nerve had a good anesthetic effect. Meanwhile, these findings confirmed that the inferior alveolar nerve in rabbits is a sensory nerve. For the animals in the control group, clear pain reactions was observed during mandibular osteotomy, indicating that anesthesia using sodium pentobarbital 
alone did not have a sufficient local analgesic effect. The injection of lidocaine in the medial mandibular ascending branch can reduce the dose of sodium pentobarbital needed and can reduce the anesthesia drug reactions. Furthermore, the injection of lidocaine in the medial mandibular ascending branch was not affected by the decreased drug concentration due to the saline flush during the mandibular osteotomy, thus extending the mandible anesthesia time, which is conducive to the smooth operation of the mandible surgery. In summary, this study investigated the anatomical characteristics of the mandible and the inferior alveolar nerve in rabbits and established an animal model of mandibular distraction osteogenesis in rabbits to verify the distribution of the inferior alveolar nerve and the effectiveness of the block anesthesia approach. However, further anatomical research is needed to understand the innervation of the medial and lateral soft tissues of the mandible.

\section{Declaration of Conflicting Interests}

None declared.

\section{Financial Disclosure}

None of the authors has a financial interest in any of the products, devices, or drugs mentioned in this manuscript.

\section{Acknowledgement}

This work was supported by the National Natural Science Foundation of China (grant number 81160130).

\section{References}

1. Zheng-Long Tang, Wen-Juan Zhang, Dong-Xiang Wang, Jia-Min Chen, Hong Ma, et al. (2014) An experimental study addressing the promotion of mandibular defect repair through the intermittent subcutaneous injection of parathyroid hormone. J Oral Maxillofac Surg 72: 419-430.

2. Cheng G, Li Z, Wan Q, Lv K, Li D, et al. (2015) A novel animal model treated with tooth extraction to repair the full-thickness defects in the mandible of rabbits. J Surg Res 194: 706-716.

3. Jiang X, Chen Y, Fan X, Zhang H, Kun L (2015) Osteogenesis and mineralization in a rabbit mandibular distraction osteogenesis model is promoted by the human LMP-1 gene. J Orthop Res 33: 521-526.

4. Kim IS, Cho TH, Lee ZH, Hwang SJ (2013) Bone regeneration by transplantation of human mesenchymal stromal cells in a rabbit mandibular distraction osteogenesis model. Tissue Eng Part A 19: 66-78.
5. Tang ZL, Bai S, Zhu PN, Li YD, Wang DX, et al. (2016) An examination of differences in the new bone formation promoted by different doses of rhPTH during mandibular distraction osteogenesis. Plast Reconstr Surg 137: 347e-354e.

6. Stübinger S, Dard M (2013) The rabbit as experimental model for research in implant dentistry and related tissue regeneration. J Invest Surg 26: 266-282.

7. Yoshimoto M, Watanabe IS, Martins MT, Salles MB, Ten Eyck GR, et al. (2009) Microstructural and ultrastructural assessment of inferior alveolar nerve damage following nerve lateralization and implant placement: An experimental study in rabbits. Int J Oral Maxillofac Implants 24: 859865.

8. Araújo AS, Fernandes AB, Maciel JV, Netto Jde N, Bolognese AM (2015) New methodology for evaluating osteoclastic activity induced by orthodontic load. J Appl Oral Sci 23: $19-25$.

9. Yu JY, Lee W, Park JH, Bayome M, Kim Y, et al. (2012) Histologic effects of intentional-socket-assisted orthodontic movement in rabbits. Korean J Orthod 42: 207-217.

10. Ogawa S, Watanabe M, Kawaai H, Tada H, Yamazaki S (2014) Lidocaine concentration in mandibular bone after subperiosteal infiltration anesthesia decreases with elevation of periosteal flap and irrigation with saline. Anesth Prog 61: 53-62.

11. Campillo VE, Langonnet $S$, Pierrefeu A, Chaux-Bodard AG (2014) Anatomic and histological study of the rabbit mandible as an experimental model for wound healing and surgical therapies. Lab Anim 48: 273-277.

12. Todd MM, Wu B, Warner DS, Maktabi M (1994) The dose-related effects of nitric oxide synthase inhibition on cerebral blood flow during isoflurane and pentobarbital anesthesia. Anesthesiology 80: 1128-1136.

13. Borkowski GL, Danneman PJ, Russell GB, Lang CM (1990) An evaluation of three intravenous anesthetic regimens in New Zealand rabbits. Lab Anim Sci 40: 270-276.

14. Gil AG, Silvan G, Illera M, Illera JC (2004) The effects of anesthesia on the clinical chemistry of New Zealand White rabbits. Contemp Top Lab Anim Sci 43: 25-29.

15. Reichert P, Rutowski R, Kielbowicz Z, Kuryszko J, Kielbowicz M (2013) General intravenous anesthesia for brachial plexus surgery in the rabbit. Pol J Vet Sci 16: 755-756.

16. Balbinotto RP, Trindade MR, Meyer FS, Muller AL, Rosa Ad Jr, et al. (2010) Anesthetic protocol for videolaparoscopic surgery in rabbits. Acta Cir Bras 25: 121-125.

17. Hexeberg E, Hexeberg S, Hessevik I, Fosse RT (1995) Midazolam in combination with fentanyl/fluanisone and nitrous oxide as anaesthesia in rabbits--cardiovascular parameters. Lab Anim 29: 400-406. 